\section{Interview with Derek Connolly}

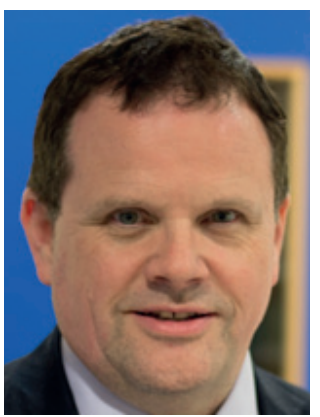

Dr Derek Connolly* speaks to Adam Price-Evans, Commissioning Editor of Future Cardiology: Derek Leslie Connolly is a Consultant Interventional Cardiologist at Birmingham City Hospital (UK). He qualified from the University of Edinburgh (UK) summa cum laude in Pharmacology in 1985 and in Medicine in 1988 where he was the Brunton Medalist. As a Carnegie scholar at the University of California, San Diego (CA, USA) he saw the early promise of angioplasty and changed his career plan from cardiac surgery to coronary intervention. He then spent a decade training in Cardiology in Cambridge (UK) where he held a British Heart Foundation PhD Fellowship at the University of Cambridge. His main clinical and research interest is the detection and treatment of atherosclerosis. He was appointed to his current role in 2000 and has been integral to the early development of both primary angioplasty and cardiac CT programs. He was UK Chief Investigator for the FOURIER trial.

First draft submitted: 5 June 2017; Accepted for publication: 5 June 2017; Published online: 16 June 2017

\section{Q Could you please begin by} introducing yourself, your background \& tell us a bit about your work to date?

I grew up in Edinburgh (UK) and undertook my first two degrees in Edinburgh medical school. I have a first class honors degree in pharmacology, so drugs have always been an interest of mine. My initial reason to go into cardiology was that when I was young, everyone in Edinburgh was dying of heart disease and there were no treatments. I thought that it would be a valid career for me to help develop treatments and hopefully reduce the level of heart disease. I then went to San Diego with the idea of being a heart surgeon and I came back as a future interventional cardiologist. I worked in Nottingham and London before working for 10 years in Cambridge, where I did a PhD in molecular cardiology. I then got a job in Birmingham in 2000 and have been here since. We are in an inner city area with a high level of deprivation and multi-ethnicity so we have high levels of heart disease; it is still the number one killer here. I've contributed to a lot of trials and with cholesterol as the biggest risk factor for heart disease, when the possibility of this new way of lowering cholesterol came in, I volunteered and became involved from the very beginning of the PCSK9 story.

\section{Q The cardiovascular field is now looking toward PCSK9 inhibition using monoclonal antibodies to lower cholesterol. Could you tell us a bit about the theory behind PCSK9 inhibition \& how it works?}

Monoclonal antibodies were invented by César Milstein and others in Cambridge (UK) and are now used in many fields, although not that much in the cardiovascular world. Around 2003, a Parisian group was trying to find the cause of very high cholesterol in families as at this time about one-third of all high cholesterol hadn't been accounted for. Their candidate gene turned out to be PCSK9 which a Canadian group had already found but didn't know it caused high cholesterol and that is when this story started. The

*Department of Cardiology, Birmingham City Hospital, Sandwell \& West Birmingham Hospitals NHS Trust, Dudley Road, Birmingham, West Midlands, B18 7QH; derek.connolly1@nhs.net

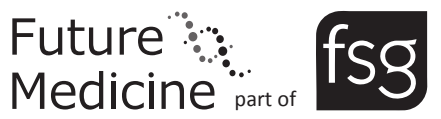

\section{KEYWORDS \\ - cholesterol • evolocumab \\ - FOURIER • monoclonal antibody \\ - PCSK9 • statin}


LDL receptor is like a little dumper truck, it sits on the surface of the liver cell and grabs bad cholesterol from the circulation, takes it in to the liver and the liver pushes it out into the gut. The LDL receptor then recycles back to the surface to do this again. PCSK9 binds the LDL receptor, such that when it is internalized by the liver, as well as getting rid of the bad cholesterol, the liver gets rid of the LDL receptor. As we know from population studies, if you have a high level of PCSK9 activity, you have a low level of LDL receptors and vice versa. There was then the discovery that there were individuals with no PCSK9 who were completely normal. This suggested that PCSK9 was responsible for modulating the LDL receptor and therefore likely to modulate LDL cholesterol in populations. Thus the idea was born that if you blocked PCSK9 you could potentially get therapeutic benefits without any side effects.

The next stage is then how do you block PCSK9? Historically we would do that with small molecule oral drugs that would bind to PCSK9 or an LDL receptor to stop the two binding. But these days, small molecules take forever to produce. The studies are being done and we may well get other oral drugs in the future to inhibit PCSK9. The advantage of monoclonal antibodies however is that you can do the technology really quickly and the company that I worked with, Amgen ${ }^{\circledR}$, have a long history of producing entirely human monoclonal antibodies. They were able to develop one quickly that would bind to PCSK9 and stop it binding to the LDL receptor and called it evolocumab. Effectively, if you give this, you don't have much circulating PCSK9, you therefore don't have LDL receptors being removed by the liver and thus LDL receptors increase. When given to normal individuals in Phase I trials, this results in a $60 \%$ reduction in $\mathrm{LDL}$ cholesterol.

Several double-blind Phase II and Phase III studies of evolocumab versus placebo were then quickly completed successfully including the GAUSS-3 studying statin intolerant patients. These showed that evolucumab seemed to reduce LDL cholesterol by a further 60\% whether you were on statins on not. On the basis of that, it got a license in 2015 in the UK and Europe. In 2016, NICE agreed we could use it in the UK in patients with higher risk and high cholesterol.
Q You were the UK chief investigator for the multinational Phase III FOURIER trial which involved the study of treatment with evolocumab in combination with statin therapy in over 27,500 patients. Could you please tell me a bit more about the trial design \& its primary goals \& objectives?

The idea of the FOURIER trial was that we wanted to do a global trial that would clearly show if evolucumab reduced the number of heart attacks and strokes. This was an enormous trial involving 27,500 patients across many countries and centers.

Although the objective of the trial was to see whether evolocumab reduced these major adverse cardiovascular events, importantly, we also conducted safety studies, as we knew that if we gave evolocumab on top of statins and ezetimibe, we'd be taking people down to the lowest levels of LDL ever seen in a clinical trial, so we wanted to confirm that was safe.

The trial design was very simple. Higher-risk patients with stable coronary disease, stroke or peripheral vascular disease were randomized to receive placebo or evolocumab via injection. All patients were already on optimal therapy depending on the country. We then followed them up every three months.

The primary composite end point was cardiovascular death, myocardial infarction (MI), stroke, hospitalization for unstable angina or coronary vascularization. The key secondary endpoints were cardiovascular death, MI or stroke. For safety, we looked at all adverse effects but particularly the sorts you see with statins, also new onset diabetes and neurocognitive effects.

\section{Q What were the results of this trial?}

We had 27,564 patients with a median follow-up of 26 months. Results showed that in patients on the evolocumab therapy, there was a $59 \%$ mean reduction in LDL cholesterol, compared with no change in the placebo group. Importantly, this reduced cholesterol level was maintained for up to 4 years in patients that continued on both statins and evolocumab therapy. This reduction in cholesterol is particularly impressive when you consider that baseline median lipid levels were not that high to begin with as this was a heavily treated group. In fact, the baseline median total cholesterol in patients was $168 \mathrm{mg} / \mathrm{dl}$ which is low when you consider that general practitioners in the UK aim to get cholesterol to below around 
$200 \mathrm{mg} / \mathrm{dl}$ following treatment. So this $59 \%$ reduction can be deemed to be impressive.

When looking at the composite primary endpoint, the results showed that cardiovascular death, MI, stroke, hospitalization for unstable angina or coronary revascularization were reduced significantly following evolocumab treatment compared with placebo. Further to this, as time continued towards 3 years followup, the benefits of evolocumab over placebo appeared to be accruing. A similar statistically significant effect was found when evaluating the secondary endpoint of cardiovascular death, MI or stroke, which showed around a $20 \%$ reduction. The growing difference between the intervention and placebo arms on primary and secondary endpoints suggest that it would be interesting to see if this benefit would continue to increase if treatment was continued past 3 years.

Together, the results showed that the main benefit of further reducing cholesterol with evolocumab, is you get around a $27 \%$ reduction in MI and a $21 \%$ reduction in stroke.

Importantly, I have never been in a drug trial before where the active intervention had numerically fewer premature discontinuations than the placebo however this happened here suggesting evolocumab was amazingly well tolerated. To see this in a clinical trial of this size was remarkable from a safety perspective.

So in summary you get $59 \%$ reduction in LDL cholesterol and easily the best side effect profile of anything I've ever seen. You can see why we are excited.

\section{Q So where do you think this leaves statins} for the treatment of high cholesterol? Do you think the additional cost of adding PCSK9 inhibition to statin therapy will be a factor in its uptake?

The first thing to say is that statins will remain, certainly for the near future, the absolute cornerstone of cholesterol reduction. This study was in addition to statins. So $70 \%$ of the patients were on what we call high intensity statins, such as atorvastatin, which actually have lower side effects than the older statins. The remaining $30 \%$ were on moderate intensity statins because some countries couldn't afford atorvastatin. In addition, $5 \%$ were on ezetimibe.

Regarding cost, you need to put it in to context. Atorvastatin was very expensive 20 years ago, but now it is cheap and the mainstay of statins. You have to look at the long game. The added competition from other new technologies will also be a factor in reducing costs. The cost of this new treatment has actually already come down and it will continue to do so. I am confident that PCSK9 is here to stay and the use will keep rising.

\section{Q What are the barriers to these new treatments?}

This is the first time in cardiovascular medicine that we have given injections long term. The big barrier that I hear is that the patients won't like the injections. In the FOURIER trial, quite the reverse seemed to be true. The patient's loved this medication because they weren't getting any side effects. The other barrier is that people in hospitals still think you can have too low an LDL cholesterol. What this study has done is put that to bed.

\section{Q What's next for PCSK9 inhibition?}

I think there are a number of exciting additional avenues. For example, using smallinterfering RNAs, my friend Professor Kausik Ray (London, UK) is developing a singleinjection technique for blocking translation of the PCSK9 mRNA and therefore PCSK9 protein production is inhibited for several months. I think small molecules are probably also coming. The other possibility is immunizing people against PCSK9, which is itself is a long term theory, but certainly the molecular tools are coming. Additionally, a second antibody-like evolocumab is on its way which will present landmark studies in the next few years. Together, all the evidence suggests that we seem to be heading in the right direction by targeting PCSK9 so it is a very exciting time.

Financial \& competing interests disclosure

$\operatorname{Dr}$ Connolly has received speaker fees and honoraria for advisory boards from Amgen. The author has no other relevant affiliations or financial involvement with any organization or entity with a financial interest in or financial conflict with the subject matter or materials discussed in the manuscript apart from those disclosed.

No writing assistance was utilized in the production of this manuscript.

\section{Disclaimer}

The opinions expressed in this interview are those of the interviewee and do not necessarily reflect the views of Future Medicine Ltd. 